\title{
Progress in molecular materials and devices
}

Initial studies on organic semiconductors began in the early 1960s in China. In the 1970s, a specialized laboratory (Lab. No. 6) was established at the Institute of Chemistry, Chinese Academy of Sciences (ICCAS), which housed the first research team engaged in this field. In 1991, Organic Solids Laboratory was formally established in ICCAS, which became the Open Laboratory, Chinese Academy of Sciences (CAS) in 1994 and re-named as CAS Key Laboratory in 2001. This team has been performing cutting-edge research on molecular materials including organic semiconductors, organic photoconductors, conducting polymers, organic conductors and superconductors, and molecular magnets. In the early 1990s, the areas of research were expanded to molecular devices with emphases on organic light-emitting diodes, organic field-effect transistors, organic solar cells, chemical/biological sensors and single-molecule devices. The Ministry of Science and Technology of China, National Natural Science Foundation of China, Chinese Academy of Sciences launched a series of development projects that significantly promoted research on molecular materials and devices. Presently, several institutes at CAS and universities are conducting fruitful research in related fields, and have recently obtained numerous influential achievements that have had a profound impact on the international science community. Currently, research from China makes an important contribution to this field.

Supported by the Chinese Chemical Society (CCS), ICCAS organized a National Symposium on Electronic Process in Organic Solids from May 16th to 18th 1991, in Beijing. The organization committee co-chaired by Prof. Renyuan Qian and Prof. Fosong Wang proposed the idea of establishing the Professional Committee of Organic Solids, which was approved at a meeting of the Standing Council of the CCS in the same year. The Professional Committee of Organic Solids has organized all of the national academic conferences in this field since then. In 2012, the Ninth National Symposium on Organic-Solids and Organic Optoelectronic Functional Materials was organized by the CCS Committee of Organic Solids, and held in Yangzhou, Jiangsu Province, China. This conference was jointly hosted by Yangzhou University and the Key Laboratory of Organic Solids, CAS. The conference concentrated on recent research achievements in the field of organic materials and devices. The conference committee invited some distinguished experts to contribute related reviews and research articles that are being published in Chinese Science Bulletin as a special issue to introduce the latest research progress made in China in this field. This special issue contains a total of 14 articles including five reviews, three letters and six research articles that cover distinct research progress in areas including the design and synthesis of conjugated molecules, molecular nanostructures, optoelectronic functions of molecular materials, carbon-based materials, theoretical study of electron transfer process, organic solar cells, and biological sensors.

Important research topics in the field of molecular materials and devices include: design and synthesis of molecular materials with good performance, study of related physical and chemical processes and basic physicochemical features, development of novel and efficient assembly technologies that are suitable to construct molecular materials and devices, and promotion of applications of molecular materials in devices, especially flexible organic devices and electronic circuits.

Currently, developed countries such as the United States, Europe, and Japan emphasize research on molecular materials and devices. Universities, research institutes, and companies have collaborated to accelerate development in this field and ultimately dominate related techniques in the future. Chinese scientists have made advances in directions including the controlled assembly of conjugated molecules, organic semiconductors with high carrier mobility, high-performance photovoltaic materials, and sensor applications, and they have accumulated extensive knowledge of related theory and mechanisms. In particular, these research projects have led to the formation of strong research teams that cooperate to undertake multidisciplinary research programs. These teams are now in an important period with the opportunity to make breakthroughs in the 
field of molecular materials. Further support is desired to promote the development of molecular materials and devices, and realize a series of technologies with proprietary intellectual property rights in our country. Ultimately, progress of this research will lead an organic electronics industry based on molecular materials and devices.

Finally, I would like to sincerely thank Mrs Li Fu, editor of Chinese Science Bulletin, and her colleagues for their support with the publication of this special issue.

ZHU DaoBen Academician of Chinese Academy of Sciences Institute of Chemistry, Chinese Academy of Sciences, Beijing 100190, China

ZHANG DeQing

Institute of Chemistry, Chinese Academy of Sciences, Beijing 100190, China

Open Access This article is distributed under the terms of the Creative Commons Attribution License which permits any use, distribution, and reproduction in any medium, provided the original author(s) and source are credited. 